\title{
Cones, Graphs and Optimal Scalings of Matrices ${ }^{\dagger}$
}

\author{
B. DAVID SAUNDERS
}

Mathematical Sciences Department, Rensselaer Polytechnic Institute, Troy, New York 12181

\section{HANS SCHNEIDER}

Mathematics Department, University of Wisconsin, Madison, Wisconsin 53706

(Received July 18, 1978)

Characterizations are given of the optimal scalings of a complex square matrix within its diagonal similarity class and its restricted diagonal equivalence class with respect to the maximum element norm. The characterizations are in terms of a finite number of products, principally circuit and diagonal products. The proofs proceed by reducing the optimal scaling problems from the multiplicative matrix level in succession to an additive matrix level, a graph theoretic level, and a geometric level involving duality theorems for cones. At the geometric level, the diagonal similarity and the restricted diagonal equivalence problems are unified.

\section{INTRODUCTION}

Let $P$ be a complex square matrix. A scaling of $P$ (by means of diagonal transformations) is a matrix $N=S P T^{-1}$, where $S, T$ are nonsingular diagonal matrices. A scaling $N$ of $P$ is optimal with respect to a given measure e.g. norm) and over a given class of scalings (e.g. all scalings with $S=T$ ) if the measure of $N$ is the minimum of measures of matrices in the class. In this paper we characterize optimal scalings with respect to the elementwise maximum norm. The classes of scalings considered will be the diagonal similarity class of $\mathbf{P}$ (viz. $S=T$ ) and the (restricted) diagonal equivalence class of $P$ (viz. det $S T^{-1}=1$ ). Our characterizations will be chiefly in terms

$\dagger$ This research was partly supported by the National Science Foundation under grants MCS76-06958, MCS 76-06374 and MCS78-01087. 
of circuit and diagonal products of $P$. Thus the optimal matrices in each class are characterized by a finite set of conditions. Some of our results are known and may be found in a variety of places in the literature, but some are new. In order to provide a unified treatment for the two classes of scalings mentioned above, we proceed on four levels:

$(M)$ There is the level of diagonal similarity and diagonal equivalence of matrices, which involves multiplication.

$(A)$ There is the additive matrix level which is obtained from $(M)$ by taking logarithms.

$(G)$ There is a graph theoretic level, involving circuits and diagonals (one-factors) of graphs associated with the pattern graphs of the matrices.

(C) There is a purely geometric level involving cones in $n$-space.

The results of each level are immediate and easy consequences of the results of the subsequent level.

In Section 2 we state at level $(M)$ two scaling problems, called by us the general and the special problem, for each of the two classes of scalings. We then formulate equivalent problems at levels $(A),(G)$ and $(C)$. At level $(C)$, the diagonal similarity and diagonal equivalence problems coincide. In Section 3, we give solutions of the problems at level $(C)$. The special problem is solved using a well-known duality theorem for polyhedral cones. The general problem is solved by a theorem related to the duality theorem for linear programming. We, however, give a simple proof of this theorem by means of the duality theorem for polyhedral cones just mentioned and the Gordan-Stiemke theorem. In Section 4 we define two pattern graphs for a matrix and we apply the two theorems of Section 3 at level $(G)$. In Section 5 we state our principal theorems which are at level $(M)$. We prove the theorems by showing the equivalence of each of their conditions to a condition found in the corresponding theorem in Section 4. An example is given in Section 6. The theorems are not explicitly stated at level $(A)$, but some of them are quoted at that level in our paper [25]. In order to identify corresponding theorems and problems at the four levels, each problem and theorem is identified by a two or three letter code explained at the beginning of Section 2.

For diagonal similarity, Afriat [1], [2], [3] has given solutions to the special scaling problem in a somewhat restricted form at level $(A)$. Independently, Fiedler-Pták [12], [13] proved some results at level $(M)$ which contain the special case, see also Engel-Schneider [10], [11]. The general case is to be found in [11]. For diagonal equivalence, our characterizations appear to be new, but results related to ours were proved by Egervary [9]. Also these problems are related to minimal cost network-flow problems, 
e.g. Ford-Fulkerson $[14, \mathrm{Ch}$. III ], see in particular the out-of-kilter algorithm found there and in Fulkerson [15]. In the two publications last mentioned, the stress is on algorithms for solving the problems, while we are concerned with finite characterizations of optimal scalings and the unifications of proofs. An algorithm for a scaling problem with respect to a measure which is not a norm may be found in Fulkerson-Wolfe [16], and characterizations of optimal scalings with respect to this measure appear in Rothblum-Schneider [23]. Results on optimal scalings with respect to a measure that involves the condition number of a matrix have appeared in Bauer [5].

It should be observed that results on the scaling of matrices have applications to other areas, in economics to demand analysis, e.g. Afriat [2] and to input-output change, e.g. Bacharach [4]. Diagonal scalings also arise in connection with the solutions of hyperbolic systems of partial differential equations, see Gunzberger-Plemmons [19].

\section{THE PROBLEMS}

The problems addressed will be identified by a two or three letter code:

(i) The levels, $(M),(A),(G),(C)$, as explained in Section 1.

(ii) $s$-special and $g$ - general.

(iii) $\sigma$-diagonal similarity and $\varepsilon$-(restricted) diagonal equivalence.

At the geometric level $(C)$, the features $\sigma$ and $\varepsilon$ are unified, and hence the third letter is not used.

We now state the optimal scaling problems at the multiplicative matrix level $(M)$. By $\mathbb{R}$ we denote the field of real numbers and by $\mathbb{C}$ the field of complex numbers. For $P \in \mathbb{C}^{n n}$, let

$$
|P|=\max _{i, j=1, \ldots, n}\left|p_{i j}\right| .
$$

We are concerned with finding the minimum value of this norm within a diagonal similarity or diagonal equivalence class, cf. [10], [11.], [24]. Hence without loss of generality we suppose that $P \in \mathbb{R}_{+}^{n n}$, where

$$
\mathbb{R}_{+}^{m n}=\left\{B \in \mathbb{R}^{n n}: \quad B \geqslant 0\right\}
$$

and all inequalities are elementwise.

Let

and let

$$
D^{n}=\left\{X \in \mathbb{R}^{n n}: \quad X \text { is nonsingular, diagonal }\right\},
$$

$$
D_{+}^{n}=\mathbb{R}_{+}^{m n} \cap D^{n} .
$$

For $P \in \mathbb{R}_{+}^{m}$, we have the following scaling problems:

$(M g \sigma)$ Find $\inf \left\{\left|S P S^{-1}\right|: S \in D_{+}^{n}\right\}$,

$(M g \varepsilon)$ Find $\quad \inf \left\{\left|S P T^{-1}\right|: S, T \in D_{+}^{n}, \operatorname{det} S T^{-1}=1\right\}$. 
The solutions to these problems will follow from the solutions of the special problems below.

Let $E \in \mathbb{R}_{+}^{n n}$ be defined by $e_{i j}=1, i, j=1, \ldots, n$.

For $P \in \mathbb{R}_{+}^{n n}$, we have

$(M S \sigma)$ Does there exist $S \in D_{+}^{n_{+}}$such that $S P S^{-1} \leqslant E$ ?

(MsE) Do there exist $S, T \in D_{+}^{n}$ with det $S T^{-1}=1$ such that $S P T^{-1} \leqslant E$ ?

By taking logarithms, we obtain problems at level $(A)$. Let $\mathbb{R}_{*}=\mathbb{R} \cup\{-\infty\}$, and let $A \in \mathbb{R}_{*}^{n n}$.

$(A g \sigma)$ Find

(AgE) Find

$$
\inf \left\{\max \left\{x_{i}+a_{i j}-x_{j}: \quad a_{i j} \neq-\infty\right\}: \quad X \in D^{n}\right\} .
$$

$$
\inf \left\{\max \left\{x_{i}+a_{i j}-y_{j}: a_{i j} \neq-\infty\right\}: \quad X, Y \in D^{n}, \operatorname{tr} X=\operatorname{tr} Y\right\}
$$

where $\operatorname{tr} B$ is the trace of $B$, for $B \in \mathbb{R}^{n n}$.

In order to deal with nonnegative quantities, we reverse the inequality and we obtain:

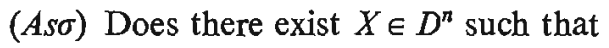

$$
x_{i}+a_{i j}-x_{j} \geqslant 0 \text { if } a_{i j} \neq-\infty \text { ? }
$$

(Ase) Do there exist $X, Y \in D^{n}$ with tr $X=\operatorname{tr} Y$, such that

$$
x_{i}+a_{i j}-x_{j} \geqslant 0 \quad \text { if } a_{i j} \neq-\infty \text { ? }
$$

We now give these additive problems graph theoretic formulations, as we did for related algebraic problems in [24]. To make this paper self-contained, we state some graph theoretic definitions. For further information on graphs see [7] or [22, Ch. 4].

\section{(2.1) Definitions}

(i) A pair $(G, V)$ is a (directed) graph if the arc set $G$ is a subset of $V \times V$, where $V$ is the vertex set.

(ii) A triple $\left(H, V, V^{\prime}\right)$ is a (directed) bipartite graph if $\left(H, V \cup V^{\prime}\right)$ is a graph for which $V \cap V^{\prime}=\varnothing$ and $H \subseteq V \times V^{\prime}$.

In this paper $V=\langle n\rangle=\{1, \ldots, n\}$ and $V^{\prime}=\langle n\rangle^{\prime}=\{n+1, \ldots, 2 n\}$, and we employ two conventions throughout:

First, we order the elements of $G$ and $H$ lexicographically. Thus e.g. $G=\left\{g_{1}, \ldots, g_{m}\right\}$, where $m=|G|$, the number of elements in $G$.

Second, we write $G$ for both the graph $(G, V)$ and the arc set $G$. Similarly identify $H$ and $\left(H, V, V^{\prime}\right)$. 
(2.2) Defintion Let $G$ be a graph. Then the (arc-vertex) incidence matrix $\Gamma$ of $G$ is the $m \times n$ matrix defined thus: $\Gamma=\left(\gamma_{r i}\right)$ where

$$
\begin{aligned}
& \gamma_{r i}=1 \quad \text { if } g_{\mathbf{r}}=(i, j) \text { and } i \neq j \text {, } \\
& \gamma_{r i}=-1 \text { if } g_{r}=(j, i) \text { and } i \neq j \text {, } \\
& \gamma_{r i}=0 \quad \text { otherwise. }
\end{aligned}
$$

Observe that $\gamma_{r i}=0$ if $g_{r}=(i, i)$. Many authors call $\Gamma^{t}$ the incidence matrix of $G$.

(2.3) Defintrions Let $\Phi_{*}$ be a set with distinguished element *, and let $A$ be an $(n \times n)$ matrix with elements in $\Phi_{*}$.

(i) The *-pattern graph for $A$ is the graph $\mathbf{G}_{*}(A)$ such that $(i, j) \in \mathbf{G}_{*}(A)$ if and only if $a_{i j} \neq *$.

(ii) The bipartite *-pattern graph for $A$ is the bipartite graph $\mathbf{H}_{*}(A)$ such that

$$
(i, j+n) \in \mathbf{H}_{*}(A) \text { if and only if } a_{i j} \neq * \text {. }
$$

(iii) Let $A, B \in \Phi_{*}^{n n}, \mathbf{G}_{*}(B) \subseteq \mathbf{G}_{*}(A)$. We define a vector $b=\eta_{A}(B) \in \Phi^{m}$ thus:

$$
b_{r}=b_{i j}, \quad \text { if } \quad g_{r}=(i, j) \in \mathbf{G}_{*}(A), \quad r=1, \ldots, m .
$$

Observe that $\eta_{A}(B)$ is also the vector given by

$$
b_{r}=b_{i j}, \quad \text { if } \quad h_{r}=(i, j+n) \in \mathbf{H}_{*}(A), \quad r=1, \ldots, m,
$$

If $B=A$, we write $\eta(A)$ for $\eta_{A}(A)$.

Let $A \in \mathbb{R}_{*}^{n n}$, where $*=-\infty$ is the distinguished element. Let $\Gamma$ and $\Delta$ be the incidence matrices of $\mathbf{G}_{*}(A)$ and $\mathbf{H}_{*}(A)$ respectively and let $a=\eta(A)$. Let $X \in D^{n}$. If $x^{t}=\left(x_{1}, \ldots, x_{n}\right)$, observe that $x_{i}-x_{j}=(\Gamma x)_{r}$, where $(i, j)=$ $g_{r} \in \mathbf{G}_{*}(A)$. If $X, Y \in D^{n}$, and $z^{t}=\left(x_{1}, \ldots, x_{n}, y_{1}, \ldots, y_{n}\right)$ then $x_{i}-y_{j}=(\Delta z)_{r}$, where $(i, j+n)=h_{r} \in \mathbf{H}_{*}(A)$. Also the condition tr $X=\operatorname{tr} Y$ corresponds to $\varphi^{t} z=0$ where $\varphi \in \mathbb{R}^{2 n}$ is defined by $\varphi_{i}=1, i \in\langle n\rangle$ and $\varphi_{i}=-1, i \in\langle n\rangle^{\prime}$.

We now state problems at level $(G)$, which correspond to the problems previously stated at level $(A)$ when $a=\eta(A), G=\mathbf{G}_{*}(A)$ and $H=\mathbf{H}_{*}(A)$.

Let $G$ be a graph and let $H$ be a bipartite graph with $|G|=|H|=m$, and incidence matrices $\Gamma \in \mathbb{R}^{m n}, \Delta \in \mathbb{R}^{m, 2 n}$ respectively. Let $a \in \mathbb{R}^{m}$.

$(G g \sigma)$ Find

$(G g \varepsilon)$ Find

$$
\begin{aligned}
& \inf _{\substack{x \in \mathbb{R}^{n} \\
\max _{r \in\langle m\rangle}}}(a+\Gamma x)_{r}, \\
& \inf _{\substack{z \in \mathbb{R}^{2 n} \\
\varphi^{t_{z}=0}}} \max _{r \in\langle m\rangle}(a+\Delta z)_{r} .
\end{aligned}
$$

(Gso) Does there exist $x \in R^{n}$ such that $(a+\Gamma x) \geqslant 0$ ?

(Gse) Does there exist $z \in \mathbb{R}^{n n}$ with $\varphi^{t} z=0$ such that $(a+\Delta z) \geqslant 0$ ?

Later we shall have occasion to discuss the spaces $W_{\sigma}$ and $W_{\varepsilon}$ defined by

$$
\begin{aligned}
& W_{\delta}=\left\{V \in \mathbb{R}^{m}: v^{t} \Gamma=0\right\}, \\
& W_{\varepsilon}=\left\{V \in \mathbb{R}^{m}: v^{t} \Delta=\lambda \varphi, \quad \text { for some } \lambda \in \mathbb{R}\right\} .
\end{aligned}
$$


Observe that

$$
\begin{aligned}
& W_{\sigma}^{\perp}=\left\{u \in \mathbb{R}^{m}: \quad \exists x \in \mathbb{R}^{n}, \quad u=\Gamma x\right\}, \\
& W_{\varepsilon}^{\perp}=\left\{u \in \mathbb{R}^{m}: \quad \exists z \in \mathbb{R}^{2 n}, \quad \varphi^{t} z=0, \quad u=\Delta z\right\} .
\end{aligned}
$$

Hence the general problems involve

$$
\inf _{u \in W^{\perp}} \max _{r \in\langle m\rangle}(a+u)_{r}
$$

for a subspace $W$ of $\mathbb{R}^{m}$. Let $\Sigma$ be the simplex

$$
\Sigma=\left\{x \in \mathbb{R}^{m}: \quad e^{t} x=1\right\},
$$

where $e=(1, \ldots, 1) \in \mathbb{R}^{m}$.

Then

$$
\max _{r \in\langle m\rangle}(a+u)_{r}=\max _{v \in \Sigma} v^{t}(a+u) .
$$

Thus appropriate versions of our problems at level $(C)$ are given by:

Let $a \in \mathbb{R}^{m}$, and let $W$ be a subspace of $\mathbb{R}^{m}$.

$(\mathrm{Cg})$ Find

$$
\inf _{u \in W^{\perp}} \max _{v \in \Sigma} v^{t}(a+u) .
$$

(Cs) Does there exist $u \in W^{\perp}$ such that $a+u \geqslant 0$ ?

An equivalent form of $(C s)$ is given by:

Is $a \in W^{\perp}+\mathbb{R}_{+}^{m}$ ? It is well-known that $W^{\perp}+\mathbb{R}_{+}^{m}$ is the dual of a pointed cone in $\mathbb{R}^{m}$. Thus in the next section we consider cones and their duals.

\section{THE GEOMIETRIC RESULTS}

We begin with some definitions. For further information see [6], [17, Ch. 2].

(3.1) DeFinitions

(i) A nonempty subset $C$ of $\mathbb{R}^{m}$ is a (convex) cone if

$$
\begin{aligned}
& x+y \in C, \text { for } x, y \in C, \\
& \lambda x \in C, \quad \text { for } \lambda \geqslant 0, \quad x \in C .
\end{aligned}
$$

(ii) A cone $C$ in $\mathbb{R}^{n}$ is pointed if $x=0$ whenever both $x \in C$ and $-x \in C$.

(iii) $A$ cone $C$ is a polyhedral cone if there exist $x^{1}, \ldots, x^{s}$ in $\mathbb{R}^{m}$ such that

$$
C=\left\{\sum_{i=1}^{s} \lambda_{i} x^{i}: \lambda_{i} \geqslant 0, \quad i=1, \ldots, s\right\} .
$$

In this case $\left\{x^{1}, \ldots, x^{s}\right\}$ is called a set of generators for $C$.

(iv) $A$ vector $x \in C$ is called an extremal in $C$ if $x=y+z$, where $y, z \in C$ implies that $y=\lambda x, z=\mu x$, where $\lambda \geqslant 0, \mu \geqslant 0$.

Observe, that $C=\{0\}$ is a pointed polyhedral cone, and 0 is an extremal. 
(3.2) Definition If $C$ is a cone in $\mathbb{R}^{m}$, the dual cone $C^{D}$ of $C$ is defined by

$$
C^{D}=\left\{y \in \mathbb{R}^{m}: y^{t} x \geqslant 0, \quad \text { for all } \quad x \in C\right\} .
$$

If $W$ is a subspace of $\mathbb{R}^{m}$, then $W^{D}=W^{\perp}$, the orthogonal complement of $W$.

The fundamental lemma is

(3.3) Lemma Let $W$ be a subspace of $\mathbb{R}^{m}$. Then

$$
\left(\mathbb{R}_{+}^{m} \cap W\right)^{D}=\mathbb{R}_{+}^{m}+W^{\perp} .
$$

Lemma (3.3) is obtained by combining Corollary (1.7) and Lemma (3.4) of Ben-Israel [6]. Alternatively, Lemma (3.3) is a consequence of Theorem 2.14, page 59 of Gale [17]. In [6] the proofs depend on a separation theorem for convex cones, in [17] they are inductive and use inequalities. As remarked in [25], there are many other papers that prove this result and Lemma (3.5) with one type of proof or the other.

(3.4) Cs-THEOREM Let $a \in \mathbb{R}^{m}$ and let $W$ be a subspace of $\mathbb{R}^{m}$. Then the following are equivalent.

(i) There exists $u \in W^{\perp}$ such that $a+u \geqslant 0$,

(ii) If $v$ is an extremal of $W \cap \mathbb{R}_{+}^{m}$ then $v^{t} a \geqslant 0$,

(iii) If $B \in \mathbb{R}^{k m}$ and $V$, the space spanned by the columns of $B^{t}$, satisfies $V \cap \mathbb{R}_{+}^{m}=W \cap \mathbb{R}_{+}^{m}$, then $B a \in B \mathbb{R}_{+}^{m}$, the cone generated by the columns of $B$.

Proof We show that each of the three conditions is equivalent to $a \in W^{\perp}+\mathbb{R}_{+}^{m}$ or $a \in\left(W \cap \mathbb{R}_{+}^{m}\right)^{D}$, which in turn are equivalent by Lemma (3.3). Condition (i) is $a \in W^{\perp}+\mathbb{B}_{+}^{m}$.

Condition (ii): Since the cone $C=W \cap \mathbb{R}_{+}^{m}$ is pointed and polyhedral [17, p. 59] there is a finite set of extremals which generate $C$ [17, p. 62]. But if $v^{t} a \geqslant 0$ for all extremals of $C$, then $v^{t} a \geqslant 0$ for all $v \in C$. It follows that (ii) is equivalent to $a \in\left(W \cap \mathbb{R}_{+}^{m}\right)^{D}$.

Condition (iii): This condition holds if and only if there is a $q \in \mathbb{R}_{+}^{m}$ such that $B a=B q$. For this $q, a-q \in V^{\perp}$ and so (iii) is equivalent to $a \in V^{\perp}+\mathbb{R}_{+}^{m}$. But since $V \cap \mathbb{R}_{+}^{m}=W \cap \mathbb{R}_{+}^{m}$, it follows by Lemma (3.3) that $V^{\perp}+\mathbb{R}_{+}^{m}=$ $W^{\perp}+\mathbb{R}_{+}^{m}$. Hence (iii) is equivalent to $a \in W^{\perp}+\mathbb{R}_{+}^{m}$.

Note that in condition (ii) we need to verify $v^{t} a \geqslant 0$ only for a finite number of extremals $v$.

(3.5) Lemma Let $W$ be a subspace of $\mathbb{R}^{m}$. Then the following are equivalent:

(i) $W \cap \mathbb{R}_{+}^{m}=\{0\}$,

(ii) $W^{\perp} \cap$ int $\mathbb{R}_{+}^{m} \neq \varnothing$.

Here int $\mathbb{R}_{+}^{m}$ is the interior of $\mathbb{R}_{+}^{m}$.

Lemma (3.5) is called the Gordan-Stiemke Theorem in [25] in view of Gordan [18] and Stiemke [26]. A more general result with a proof depending on a separation theorem is Nikaido [21, Theorem 3.6]. For a proof of 
Lemma (3.5) depending on inequalities see the corollary on page 48 of Gale [17].

We recall that $\Sigma=\left\{x \in \mathbb{R}_{+}^{m}: \quad \sum_{i=1}^{m} x_{i}=1\right\}$.

(3.6) Cg-Theorem Let $W$ be a subspace of $\mathbb{R}^{m}$ and let $a \in \mathbb{R}^{m}$.

(i) If $W \cap \mathbb{R}_{+}^{m}=\{0\}$, then

$$
\inf _{u \in W^{\perp}} \max _{v \in \Sigma} v^{t}(a+u)=-\infty .
$$

(ii) If $W \cap \mathbb{R}_{+}^{m} \neq\{0\}$, then

$$
\min _{u \in W^{\perp}} \max _{v \in \Sigma} v^{t}(a+u)=\max _{v \in \Sigma \cap W} v^{t} a .
$$

(We have written min to show that the infimum is achieved in this case).

Proof (i) If $W \cap \mathbb{R}_{+}^{m}=\{0\}$, then by Lemma (3.5), there exists, $u \in W^{\perp}$ with $-u>0$. So we can find $\lambda>0$ such that

$$
\max _{v \in \Sigma} v^{t}(a+\lambda u)=\max _{r \in\langle m\rangle}(a+\lambda u)_{r}
$$

is arbitrarily small. The result follows.

(ii) Suppose $W \cap \mathbb{R}_{+}^{m} \neq\{0\}$. Then $W \cap \Sigma \neq \varnothing$ and since $W \cap \Sigma$ is a bounded polyhedral subset of $\mathbb{R}_{+}^{m}, \max _{v \in \Sigma \cap W} v^{t} a$ exists and is achieved. Let

Let $u \in W^{\perp}$. Then

$$
\eta \equiv \max _{v \in \Sigma \cap W} v^{t} a
$$

$$
\max _{v \in \Sigma} v^{t}(a+u) \geqslant \max _{v \in \Sigma \cap W} v^{t}(a+u)=\max _{v \in \Sigma \cap W} v^{t} a=\eta .
$$

On the other hand, $v^{t}(\eta e-a) \geqslant 0$ for all $v \in \Sigma \cap W$. Hence, by Theorem (3.4) there is a $u_{0} \in W^{\perp}$ such that $\eta e-a-u_{0} \geqslant 0$, and so $a+u_{0} \leqslant \eta e$. Then $v^{t}\left(a+u_{0}\right) \leqslant \eta$, for all $v \in \Sigma$, and we obtain that $\max _{v \in \Sigma} v^{t}\left(a+u_{0}\right) \leqslant \eta$. The result follows.

Theorem (3.6) may also be derived from the duality theorem of linear programming, e.g. Gale [17, Ch. 3]. For a related result derived in this way, see [23].

\section{APPIICATIONS TO GRAPHS}

We require some additional concepts, cf. [7], [22]. A $\{0,1\}$-vector in $\mathbb{R}^{m}$ is a vector $v \in \mathbb{R}^{m}$ such that $v_{\boldsymbol{r}}=0$ or $v_{\boldsymbol{r}}=1$, for $r \in\langle m\rangle$. A $\{0,1,-1\}$ vector is defined similarly.

(4.1) DeFInITrons

(i) Let $G$ be a graph, and let $\Gamma \in \mathbb{R}^{m n}$ be its incidence matrix. $A$ cycle for $G$ is a $\{0,1,-1\}$-vector in $\mathbb{R}^{n}$ such that $v^{t} \Gamma=0 . A$ cycle $v$ for $G$ is a (simple) 
circuit for $G$ if $v$ is $a\{0,1\}$-vector which is not the sum of two $\{0,1\}$-cycles for $G$.

(ii) Let $H$ be a bipartite graph and let $\Delta \in \mathbb{R}^{m, 2 n}$ be its incidence matrix. $A$ pseudo-diagonal is a vector $v \in \mathbb{R}^{m}$ such that $v^{t} \Delta=\varphi^{t} A$ diagonal (onefactor) for $H$ is a $\{0,1\}$-pseudo-diagonal.

We shall use the terms cycle space $W_{\sigma}$, circuit space $V_{\sigma}$, pseudo-diagonal space $W_{\varepsilon}$, and diagonal space $V_{\varepsilon}$ for the subspaces of $\mathbb{R}^{m}$ spanned by the corresponding objects. It follows from some elementary graph theory that $W_{\sigma}=\left\{v \in \mathbb{R}^{m}: v^{t} \Gamma=0\right\}$, cf. [22, p. 396], which is the space defined near the end of Section 2. It is elementary linear algebra that $W_{\varepsilon}=\left\{v \in \mathbb{R}^{m}\right.$ : $\left.v^{t} \Delta=\lambda \varphi, \lambda \in \mathbb{R}\right\}$, which is again the space defined previously.

We also define $C_{\sigma}=V_{\sigma} \cap \mathbb{R}_{+}^{m}$. It is known, [7, p. 91] that every nonnegative element of $W_{\sigma}$ is a linear combination with positive coefficient of circuits for $G$. Hence $C_{\sigma}=W_{\delta} \cap \mathbb{R}_{+}^{m}$, and the extremals of $C_{\sigma}$ are precisely the nonnegative multiples of the circuits for $G$. We define $C_{\varepsilon}=V_{\varepsilon} \cap \mathbb{R}_{+}^{m}$. It is known that every nonnegative element of $W_{e}$, is a linear combination with positive coefficients of diagonals for $H$. (This is essentially the wellknown Birkhoff-König Theorem, [8], [20], see [25] for details and further references. Hence $C_{\varepsilon}=W_{\varepsilon} \cap \mathbb{R}_{+}^{m}$, and the extremals of $C_{\varepsilon}$ are precisely the nonnegative multiples of diagonals for $H$. Note that it is possible that $V_{\sigma} \varsubsetneqq W_{\sigma}$ and $V_{\varepsilon} \subsetneq W_{\varepsilon}$.

(4.2) Gso-THeOReM Let $G$ be a graph, let $\Gamma$ be its incidence matrix, and let $a \in \mathbb{R}^{m}$. The following are equivalent.

(i) There exists $x \in \mathbb{R}^{n}$ such that $a+\Gamma x \geqslant 0$,

(ii) For all circuits $v$ of $G, v^{t} a \geqslant 0$,

(iii) If $B \in \mathbb{R}^{k m}$ and the columns of $B^{t}$ span the cycle space or the circuit space for $G$, then $B a \in B \mathbb{R}_{+}^{m}$, the cone generated by the columns of $B$.

Proof Apply the Cs-Theorem (3.4) with $W=W_{\sigma}$ and $V=W_{\sigma}$ or $V=V_{\sigma}$.

(4.3) Gse-THEOREM Let $H$ be a bipartite graph, let $\Delta \in \mathbb{R}^{m, 2 n}$ be its incidence matrix, and let $a \in \mathbb{R}^{m}$. The following are equivalent.

(i) There exists $z \in \mathbb{R}^{2 n}$ with $\varphi^{x} z=0$ such that $a+\Delta z \geqslant 0$.

(ii) For all diagonals $v$ of $H, v^{t} a \geqslant 0$.

(iii) If $B \in \mathbb{R}^{k m}$ and the columns of $B^{t}$ span the pseudo-diagonal space or the diagonal space for $H$, then $B a \in B \mathbb{R}_{+}^{m}$.

Proof Apply Cs-Theorem (3.4) with $W=W_{\varepsilon}$ and $V=W_{\varepsilon}$ or $V=V_{\varepsilon}$. (4.4) Ggo-Theorem Let $G$ be a graph, let $\Gamma \in \mathbb{R}^{m n}$ be its incidence matrix. Let $a \in \mathbb{R}^{m}$. 
(i) If there are no circuits for $G$, then

$$
\inf _{x \in \mathbb{R}^{n}} \max _{r \in\langle m\rangle}(a+\Gamma x)_{r}=-\infty .
$$

(ii) If there are circuits for $G$ then

$$
\min _{x \in \mathbb{R}^{m}} \max _{r \in\langle m\rangle}(a+\Gamma x)_{r}=\max \left\{v^{t} a / v^{t} e: v \text { is a circuit for } G\right\} \text {. }
$$

Proof Apply $\mathrm{Cg}$-Theorem (3.6) with $W=W_{\sigma}$, noting that $v / v^{t} e \in \Sigma \cap W_{\sigma}$, if $v$ is a circuit for $G$.

(4.5) Gge-THEOREM Let $H$ be a bipartite graph, and let $\Delta \in \mathbb{B}^{m, 2 n}$ be its incidence matrix. Let $a \in \mathbb{R}^{m}$.

(i) If there are no diagonals for $H$, then

$$
\inf _{\substack{z \in \mathbb{R}^{2 n} \\ \varphi^{2} \boldsymbol{m}_{z}=0}} \max _{r \in\langle n\rangle}(a+\Delta z)_{r}=-\infty .
$$

(ii) If there are diagonals for $H$, then

$$
\min _{\substack{z \in \mathbb{R}^{2 n} \\ \varphi^{\prime} z=0}} \max _{r \in\langle m\rangle}(a+\Delta z)_{r}=\frac{1}{n} \max \left\{v^{t} a: v \text { is a diagonal for } H\right\} .
$$

Proof Apply the $C g$-Theorem (3.6) with $W=W_{z}$, noting that $v / n \in$ $S \cap W_{R}$, if $v$ is a diagonal for $H$.

Many equivalent conditions to the hypotheses in (i) and (ii) of Theorems (4.4) and (4.5) are known, e.g. [25].

\section{MULTIPLICATIVE SCALING THEOREMS FOR MATRICES}

In this section we state our main results, which are at level $(M)$. We apply Definition (2.3) with $\Phi_{*}=\mathbb{R}_{*}$ and $*=0$. Let $P \in \mathbb{R}_{+}^{\prime n}$ and let $V \in \mathbb{R}_{+}^{n n}$ where $\mathbf{G}_{0}(V) \subseteq \mathbf{G}_{0}(P)$ (or, equivalently, $\mathbf{H}_{0}(V) \subseteq \mathbf{H}_{0}(P)$ ). We define

$$
\Pi_{V}(P)=\Pi_{p_{i j} \neq 0} p_{i j}^{v_{i j}} .
$$

Alternatively, suppose $v \in \mathbb{R}^{m}$. Then we may put

$$
\Pi_{v}(P)=\prod_{\mathbf{r} \in\langle m\rangle} p_{\boldsymbol{r}}^{v_{r}}
$$

where $p=\eta(P)$ and $\eta$ is as defined in (2.3-iii). Clearly (5.1) and (5.2) coincide if $\mathbf{G}_{0}(V) \subseteq \mathbf{G}_{0}(P)$ and $v=\eta_{P}(V)$. If $v$ is a circuit for $\mathbf{G}_{0}(P)$, then

$$
\Pi_{v}(P)=p_{i_{1} i_{2}} \ldots p_{i_{k-1} i_{k}} p_{i_{k} i_{1}},
$$

for distinct integers $\left(i_{1}, \ldots, i_{k}\right), k \geqslant 1, i_{r} \in\langle n\rangle, r=1, \ldots, k$. If $v$ is a diagonal for $\mathbf{H}_{0}(P)$, then

$$
\Pi_{v}(P)=p_{1 \sigma(1)} \ldots p_{n \sigma(n)}
$$

where $\sigma$ is a permutation of $(1, \ldots, n)$. 
Proofs of the four theorems in this section are obtained by reducing each condition to a condition at level $(A)$ and then to a condition at level $(G)$, which has the same number in the corresponding theorem, e.g. (i) in the $M s \sigma$-Theorem corresponds to (i) in the $G s \sigma$-Theorem. For this purpose we introduce the following notation: if $S \in D_{+}^{n}$, then $\tilde{s} \in \mathbb{R}^{n}$ is given by $\tilde{s}_{i}=\log s_{i}, i=1, \ldots, n$. If $P \in \mathbb{R}_{+}^{m n}$, then $\tilde{p} \in \mathbb{R}^{m}$ is defined by $\tilde{p}_{r}=\log p_{i j}$, for $g_{r}=(i, j) \in \mathbf{G}_{0}(P)$. The reduction will be carried out in detail in the case of the $M s \sigma$-Theorem.

(5.2) Ms $\sigma$-THEOREM Let $P \in \mathbb{R}_{+}^{n n}$. Then the following are equivalent:

(i) There exists $S \in D_{+}^{n}$ such that $S P S^{-1} \leqslant E$.

(ii) For all circuits $v$ of $\mathbf{G}_{0}(P), \Pi_{v}(P) \leqslant 1$.

(iii) Let $v^{1}, \ldots, v^{k}$ be a basis for the circuit space (or cycle space) of $\mathbf{G}_{0}(P)$. There exists a matrix $Q \in \mathbb{R}_{+}^{n n}$ with $\mathbf{G}_{0}(Q)=\mathbf{G}_{0}(P)$ such that $Q \leqslant E$ and $\Pi_{v}(P)=\Pi_{v}(Q)$, if $v \in\left\{v^{1}, \ldots, v^{k}\right\}$.

Proof (i) In the notation introduced before the statement of the theorem $S P S^{-1} \leqslant E$, where $S \in D_{+}^{n}$, is equivalent to

$$
\tilde{s}_{i}+\tilde{p}_{i j}-\tilde{s}_{j} \leqslant 0 \quad \text { if } \quad(i, j) \in \mathbf{G}_{0}(P) .
$$

But as explained in Section 2, this is equivalent to

$$
\tilde{p}+\Gamma \tilde{s} \leqslant 0,
$$

where $\Gamma$ is the incidence matrix for $\mathbf{G}_{0}(P)$. Hence (i) is identical to (i) of the Gs $\sigma$-Theorem (4.2) provided that $G=\mathbf{G}_{0}(P), a=-\tilde{p}$, and $x=-\tilde{s}$.

(ii) Let $v$ be a circuit for $\mathbf{G}_{0}(P)$. Then $\Pi_{v}(P) \leqslant 1$ if and only if

$$
\sum_{(i, j) \in G_{0}(P)} \tilde{p}_{i j} \leqslant 0 .
$$

But this, in turn, is equivalent to $v^{t} \tilde{p} \leqslant 0$, and hence (ii) becomes condition (ii) in the Gso-Theorem (4.2) with $G=\mathbf{G}_{0}(P)$ and $a=-\tilde{p}$.

(iii) Let $v \in\left\{v^{1}, \ldots, v^{k}\right\}$, and let $Q \in \mathbb{R}^{n n}$, with $\mathbf{G}_{0}(Q)=\mathbf{G}_{0}(P)$. Then $P \leqslant E$ is equivalent to $0 \leqslant-\tilde{q} \in \mathbb{R}^{m}$, and $\Pi_{v}(P)=\Pi_{v}(Q)$ is equivalent to $v^{t} \tilde{p}=v^{z} \tilde{q}$. Thus (iii) becomes (iii) of (Gso)-Theorem (4.2) with $a=-\tilde{p}$, $G=\mathbf{G}_{0}(P)$ and $B^{t}$ the matrix whose columns are $v^{1}, \ldots, v^{k}$.

The equivalence of conditions (i) and (ii) of Theorem (5.2) for the case that $\mathbf{G}_{0}(P)$ is the full (directed) graph with vertex set $\langle n\rangle$, is found in Afriat $[1,2,3]$ at level $(A)$. Fiedler-Pták [12, 13] and Engel-Schneider $[10,11]$ also proved this equivalence. The equivalence of condition (5.2-iii) to the others may be new. We now make four remarks on Theorem (5.2).

(5.3) Remark In Theorem (5.2-iii), $v^{1}, \ldots, v^{k}$ need not be linearly independent. It suffices that $v^{1}, \ldots, v^{k}$ span a space which contains the circuit space for $\mathbf{G}_{0}(P)$ and which is contained in the cycle space for $\mathbf{G}_{0}(P)$. Further, the condition $\mathbf{G}_{0}(Q)=\mathbf{G}_{0}(P)$ on $Q$ may be relaxed, for (iii) $\Rightarrow$ (i)remains valid provided that $\mathbf{G}_{0}(P) \subseteq \mathbf{G}_{0}(Q)$. 
(5.4) Remark Condition (5.2-ii) is equivalent to : (ii). For all sequences of distinct integers $\left(i_{1}, \ldots, i_{k}\right)$ in $\langle n\rangle$ and $i_{k+1}=i_{1}$,

$$
\prod_{r \in\langle k\rangle} p_{i_{r} i_{r+1}} \leqslant 1 .
$$

These products correspond to all circuits of the complete directed graph on the vertex set $\langle n\rangle$. But it is not possible in (5.2-iii) to use products corresponding to a basis for the circuit space of this graph, as the following example shows.

Example Let $n=2$. Then the products $p_{22}, p_{11} p_{22}, p_{12} p_{21}$ correspond to a basis for the circuit or cycle space of the complete graph. Let

$$
P=\left[\begin{array}{ll}
2 & 1 \\
1 & 0
\end{array}\right] \quad Q=\left[\begin{array}{ll}
1 & 1 \\
1 & 0
\end{array}\right] .
$$

Then $q_{22}=p_{22}, q_{11} q_{22}=p_{11} p_{22}, q_{12} q_{21}=p_{12} p_{21}$, yet $P$ does not satisfy (5.2-ii), since $p_{11}>1$.

(5.5) Remark If $v^{1}, \ldots, v^{k}$ is a basis for the circuit space of $\mathbf{G}_{0}(P)$, then (5.2-iii) may be satisfied, yet $P$ may not be diagonally similar to $Q$, cf. [24] for definition. For suppose that

$$
P=\left[\begin{array}{ccc}
0 & p_{12} & p_{13} \\
0 & 0 & p_{23} \\
0 & 0 & 0
\end{array}\right],
$$

where $p_{12}, p_{13}$ and $p_{23}$ are nonzero. In this case, the circuit space of $\mathbf{G}_{0}(P)$ is $\{0\}$. Hence any $Q \in \mathbb{R}^{33}$ such that $\mathbf{G}_{0}(Q)=\mathbf{G}_{0}(P)$ satisfies (5.2-iii). But for diagonal similarity with $P$ we require $q_{12} q_{13} q_{23}^{-1}=p_{12} p_{13} p_{23}^{-1}$, cf. [24].

(5.6) Remark Let $P, P^{\prime} \in \mathbb{R}_{+}^{n n}$, where $\mathbf{G}_{0}(P)=\mathbf{G}_{0}\left(P^{\prime}\right)$. Theorem (5.2) easily generalizes to yield necessary and sufficient conditions for the existence of $S \in D_{+}^{n}$ such that $S P S^{-1} \leqslant P^{\prime}$. For let $P^{\prime \prime} \in \mathbb{R}_{+}^{n n}$ be defined by $p_{i j}^{\prime \prime}=p_{i j} / p_{i j}^{\prime}$, for $(i, j) \in \mathbf{G}_{0}(P)$ and $p_{i j}^{\prime \prime}=0$, otherwise. Then $S P S^{-1} \leqslant P^{\prime}$ is clearly equivalent to $S P^{\prime \prime} S^{-1} \leqslant E$. Thus condition (5.2-ii) is replaced by $\Pi_{v}(P) \leqslant$ $\Pi_{v}\left(P^{\prime}\right)$ for all circuits $v$ of $\mathbf{G}_{0}(P)$. See [13, Theorem 3.5] for a result with an additional hypothesis on $P$, but where $P, P^{\prime} \in \mathbb{C}^{n n}$ is permitted. If $v^{1}, \ldots, v^{k}$ are chosen as in Theorem (5.2), condition (5.2-iii) is replaced by: There exists $Q \in \mathbb{R}_{+}^{n n}$, with $\mathbf{G}_{0}(Q)=\mathbf{G}_{0}(P)$ such that $\Pi_{v}(P)=\Pi_{v}\left(Q \circ P^{\prime}\right)$ for $v \in\left\{v_{1}, \ldots, v_{k}\right\}$, where $Q \circ P^{\prime}$ is the Hadamard (elementwise) product of $Q$ and $P^{\prime}$.

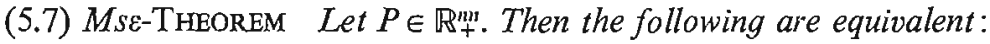

(i) There exist $S, T \in D_{+}^{n}$ with $\operatorname{det} S T^{-1}=1$ such that $S P T^{-1} \leqslant E$.

(ii) For all diagonals $v$ of $\mathbf{H}_{0}(P), \Pi_{v}(P) \leqslant 1$.

(iii) Let $v^{1}, \ldots, v^{k}$ be a basis for the diagonal space (or the pseudo-diagonal space) of $\mathbf{H}_{0}(P)$. Then there exists a matrix $Q \in \mathbb{R}_{+}^{n n}$ with $\mathbf{H}_{0}(P)=\mathbf{H}_{0}(Q)$ such that $Q \leqslant E$ and $\Pi_{v}(Q)=\Pi_{v}(P)$, if $v \in\left\{v^{1}, \ldots, v^{k}\right\}$. 
Proof Similar to that of Theorem (5.2).

Comments corresponding to (5.3)-(5.6) apply to Theorem (5.7).

(5.8) $M g \sigma$-THEorem Let $P \in \mathbb{R}_{+}^{\text {nn }}$.

i) If there are no circuits for $\mathbf{G}_{0}(P)$, then

$$
\inf \left\{\left|S P S^{-1}\right|: \quad S \in D_{+}^{n}\right\}=0 .
$$

ii) If there are circuits for $\mathbf{G}_{0}(P)$, then

$\min \left\{\left|S P S^{-1}\right|: \quad S \in D_{+}^{n}\right\}=\max \left\{\Pi_{v}(P)^{1 / v^{\mathrm{t} e}}: \quad v\right.$ is a circuit for $\left.\mathbf{G}_{0}(P)\right\}$.

Proof Reversing the steps in Section 2 we see that, for $S \in D_{+}^{n}$,

$$
\log \left|S P S^{-1}\right|=\max _{r \in\langle m\rangle}(a+\Gamma x)_{r},
$$

where $\Gamma$ is the incidence matrix of $\mathbf{G}_{0}(P), a=\tilde{p}$ and $x=\tilde{s}$. Also $\log \Pi_{v}(P)^{1 / v^{t} e}=v^{t} a / v^{t} e$. The theorem now follows from the $G g \sigma$-Theorem (4.4).

Theorem (5.8) is essentially Theorem 7.2 and Remark 7.3 of [11].

(5.9) $M g \varepsilon$-THEOREM Let $P \in \mathbb{R}_{+}^{n n}$.

i) If there are no diagonals for $\mathbf{H}_{0}(P)$, then

$$
\inf \left\{\left|S P T^{-1}\right|: \quad S, T \in D_{+}^{n}, \operatorname{det} S T^{-1}=1\right\}=0 .
$$

ii) If there are diagonals for $\mathbf{H}_{0}(P)$, then

$$
\begin{aligned}
& \inf \left\{\left|S P T^{-1}\right|: \quad S, T \in D_{+}^{n}, \operatorname{det} S T^{-1}=1\right\} \\
& \quad=\max \left\{\Pi_{v}(P)^{1 / n}: \quad v \text { is a diagonal for } \mathbf{H}_{0}(P)\right\} .
\end{aligned}
$$

Proof Similar to $M g \sigma$-Theorem (5.8).

\section{EXAMPLE}

Let

Then $\mathbf{H}_{0}(P)$ is

$$
P=\left[\begin{array}{llll}
\frac{1}{2} & \frac{1}{4} & 0 & 0 \\
2 & 2 & 0 & 0 \\
0 & 0 & \frac{1}{4} & 1 \\
0 & 0 & \frac{1}{2} & 4
\end{array}\right]
$$
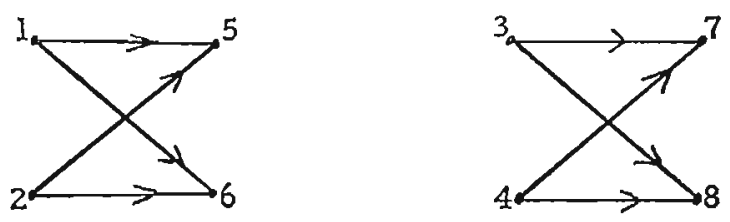
There are four diagonals for $\mathbf{H}_{0}(P)$ and the corresponding products are $\frac{1}{4}, \frac{1}{2}, \frac{1}{2}, 1$. Hence, by condition (5.7-ii), there exist $S, T \in D_{+}^{n}$ with $\operatorname{det} S T^{-1}=$ 1 such that $S P T^{-1} \leqslant E$. It is possible to reach this conclusion by checking just three products, if we use condition (5.7-iii). For, a basis for the diagonal space of $\mathbf{H}_{0}(A)$ is given by the columns of $B$, where

$$
B^{t}=\left[\begin{array}{rrrrrrrr}
1 & 0 & 0 & 1 & 1 & 0 & 0 & 1 \\
1 & -1 & -1 & 1 & 0 & 0 & 0 & 0 \\
0 & 0 & 0 & 0 & 1 & -1 & -1 & 1
\end{array}\right]
$$

Here the first row of $B^{t}$ corresponds to the identity matrix and the others to polygon matrices, cf. [24]. For $\tilde{p}_{r}=\log _{2} p_{i j}$, if $h_{r}=(i, j) \in \mathbf{H}_{0}(P)$ we have

and hence

$$
-\tilde{p}^{t}=\left[\begin{array}{llllllll}
1 & 2 & -1 & -1 & 2 & 0 & 1 & -2
\end{array}\right]
$$

where

$$
(-B \tilde{p})^{t}=\left[\begin{array}{lll}
0 & -1 & -1
\end{array}\right]=(-B \tilde{r})^{t}
$$

$$
-\tilde{r}^{t}=\left[\begin{array}{llllllll}
0 & 1 & 0 & 0 & 0 & 1 & 0 & 0
\end{array}\right] \geqslant 0 .
$$

Hence condition (5.6-iii) holds, with

By Theorem 5.7,

$$
Q=\left[\begin{array}{llll}
1 & \frac{1}{2} & 0 & 0 \\
1 & 1 & 0 & 0 \\
0 & 0 & 1 & \frac{1}{2} \\
0 & 0 & 1 & 1
\end{array}\right]
$$

$$
\min \left\{\left|S P T^{-1}\right|: \quad S, T \in D_{+}^{n}, \operatorname{det} S T^{-1}=1\right\}=1 .
$$

\section{References}

[1] S. N. Afriat, The system of inequalities $a_{r s}>X_{r}-X_{s}$, Proc. Cambridge Phil. Soc. 59 (1963), 125-133.

[2] S. N. Afriat, On a system of inequalities in demand analysis: an extension of the classical method, Intern. Econ. Rev. 14 (1973), 460-472.

[3] S. N. Afriat, On sum-symmetric matrices, Lin. Alg. Appl. 8 (1974), 129-140.

[4] M. Bacharach, Biproportional Matrices in Input-Output Change, Cambridge U.P. (1970); Cambridge Univ. Dept. App. Econ. Mono. 16.

[5] F. L. Bauer, Optimally scaled matrices, Num. Math. 5 (1963), 73-87.

[6] A. Ben-Israel, Linear equations on finite dimensional, real or complex, vector spaces; a unified theory, J. Math. Anal. Apps. 27 (1969), 367-399.

[7] C. Berge, Graphs and Hypergraphs, North-Holland, Amsterdam (1973).

[8] G. Birkhoff, Tres observationes sobre el algebra lineal, Univ. Nac. Tucuman Rev., Ser A.5 (1946), 147-150.

[9] E. Egerváry, On combinatorial properties of matrices, Mat. Fiz. Lapok 38 (1931), 16-28 and G. Washington Log. Pap. 11 (1955).

[10] G. M. Engel and H. Schneider, Cyclic and diagonal products on a matrix, Lin. Alg. Appl. 7 (1973), 301-335.

[11] G. M. Engel and H. Schneider, Diagonal similarity and equivalence for matrices over groups with 0, Czech Math. J. 25 (100) (1975), 389-403. 
[12] M. Fiedler and V. Pták, Diagonally dominant matrices, Czech. M. J. 17 (92) (1967), $420-433$.

[13] M. Fiedler and V. Pták, Cyclic products and an inequality for determinants, $C_{z e c h}$. Math. J. 19 (94) (1969), 428-451.

[14] L. R. Ford and D. R. Fulkerson, Flows in Netwosks, Princeton U.P. (1962).

[15] D. R. Fulkerson, An out-of-kilter method for minimal-cost flow problems, SIAM J. 9 (1961), 18-27.

[16] D. R. Fulkerson and P. Wolfe, An algorithm for scaling matrices, SIAM Rev. 4 (1962), 142-146.

[17] D. Gale, The Theory of Linear Economic Models, McGraw-Hill, New York (1960).

[18] P. Gordan, Ueber die Auflösung linearer Gleichungen mit reellen Coefficienten, Math. Ann. 6 (1873), 23-28.

[19] M. D. Gunzberger and R. J. Plemmons, Energy conserving norms for the solution of hyperbolic systems of partial differential equations (to appear).

[20] D. König, Über Graphen and ihre Anwendung auf Determinantentheorie und Mengenlehre, Math. Ann. 77 (1916), 453-465.

[21] H. Nikaido, Convex Structures and Economic Theory, Academic Press, New York (1968).

[22] M. Pearl, Matrix Theory and Finite Mathematics, McGraw-Hill, New York (1973).

[23] U. G. Rothblum and H. Schneider, Characterizations of optimal scalings of matrices, Math. Prog. (to appear).

[24] B. D. Saunders and H. Schneider, Flows on graphs applied to diagonal similarity and diagonal equivalence for matrices, Discrete Math. 24 (1978), 205-220.

[25] B. D. Saunders and H. Schneider, Applications of the Gordan-Stiemke Theorem in combinatorial matrix theory, SIAM Rev. 21 (1979), 528-541.

[26] E. Stiemke, Über positive Lösungen homogener linearer Gleichungen, Math. Ann. 76 (1915), 340-342. 